\title{
Free energy and vibrational entropy difference between ordered and disordered $\mathrm{Ni}_{3} \mathrm{Al}$
}

\author{
R. Ravelo* \\ Physics Department and Materials Research Institute, University of Texas, El Paso, Texas 79968 \\ J. Aguilar \\ Materials Research Institute, University of Texas, El Paso, Texas 79968 \\ M. Baskes and J. E. Angelo ${ }^{\dagger}$ \\ Materials Reliability Department, Sandia National Laboratories, Livermore, California 94551 \\ B. Fultz \\ Division of Engineering and Applied Science, 138-78, California Institute of Technology, Pasadena, California 91125 \\ Brad Lee Holian \\ Theoretical Division, Los Alamos National Laboratory, Los Alamos, New Mexico 87545
}

(Received 17 October 1996; revised manuscript received 15 May 1997)

\begin{abstract}
We have calculated free energy and vibrational entropy differences in $\mathrm{Ni}_{3} \mathrm{Al}$ between its equilibrium ordered structure and a disordered fcc solid solution. The free energy and entropy differences were calculated using the method of adiabatic switching in a molecular-dynamics formalism. The path chosen for the free-energy calculations directly connects the disordered with the ordered state. The atomic interactions are described by embedded-atom-method potentials. We find that the vibrational entropy difference increases with temperature from $0.14 k_{B}$ /atom at $300 \mathrm{~K}$ to $0.22 k_{B}$ /atom at $1200 \mathrm{~K}$. We have calculated the density of states (DOS) of the disordered phase from the Fourier transform of the velocity-velocity autocorrelation function. The disordered DOS looks more like a broadened version of the ordered DOS. Analysis of the partial density of states shows that the $\mathrm{Al}$ atoms vibrations are most affected by the compositional disorder. The phonon partial spectral intensities along the $\langle 100\rangle$ direction show that the vibrational spectrum of the disordered phase contains intensities at optical mode frequencies of the ordered alloy. We find that the volume difference between the ordered and disordered phases plays the most crucial role in the magnitude of the vibrational entropy difference. If the lattice constant of the two phases is set to the same value, the vibrational entropy difference decreases to zero. [S0163-1829(98)06202-X]
\end{abstract}

\section{INTRODUCTION}

Recently, there has been considerable interest in the vibrational contribution to the entropy of alloys. ${ }^{1-5}$ It has been known for some time that for concentrated alloys and liquid solutions, the entropy of mixing is somewhat larger than calculated configurational entropy values. ${ }^{6}$ However, there is little experimental evidence as to the contribution of vibrational entropy to order-disorder transformations in alloys. ${ }^{7,8}$

In the case of $\mathrm{Ni}_{3} \mathrm{Al}$, the $L 1_{2}$ phase is known to remain ordered up to very near its melting point $\left(1385^{\circ} \mathrm{C}\right)$. Recent experiments have estimated the vibrational entropy difference between the equilibrium $L 1_{2}$ ordered phase and the disordered fcc solid solution phase (this phase will be called henceforth the disordered phase) to be a large fraction of the configurational entropy difference $\left(0.56 k_{B} /\right.$ atom, $k_{B}$ $=$ Boltzmann constant). ${ }^{3}$ Low-temperature calorimetry experiments have estimated the vibrational entropy to be $0.3 k_{B}$ atom $^{2}$ while more recent neutron-scattering experiments place this number at $0.2 k_{B}$ /atom. ${ }^{5}$ Although the origin of this large difference is not completely well understood, it is associated with changes in the optical modes involving motion of the light $\mathrm{Al}$ atoms.

There have been few theoretical studies of vibrational en- tropy differences between ordered and compositionally disordered phases. In the ordered phase, the vibrational entropy is usually obtained via the density of states (DOS) using the harmonic or quasiharmonic approximation. ${ }^{9}$ For disordered systems, a popular method in calculations of vibrational entropies is the "virtual-crystal" method. ${ }^{10,11}$ In this method, the fully disordered system is replaced by an equivalent ordered monoatomic system with masses and interactions defined by the stoichiometry of the ordered system. This method which is in fact a perturbative approach assumes that the mass difference between the species is small. Inelastic neutron-scattering experiments of disordered $\mathrm{Ni}_{3} \mathrm{Al}$ have found intensities at optical mode frequencies in the vibrational spectrum of the disordered phase. ${ }^{5}$ This finding has been used to argue that the vibrational spectrum of the disordered system must be different from that of a monoatomic fcc crystal and therefore there might exist problems in the analysis of the phonon DOS with the virtual-crystal approximation. ${ }^{5}$

Another way of calculating the vibrational entropy is to apply lattice dynamics to the disordered system. Employing this method, Ackland calculated the vibrational entropy difference by treating the disordered phase as a superstructure with a 256-atom unit cell. ${ }^{4}$ The system is compositionally 
disordered within the superperiodicity. The main drawback of the method is the very large dynamical matrices that need to be solved in order to obtain an accurate phonon density of state (DOS) of the disordered system. Even on a supercomputer, memory and time limitations prevent DOS calculations with systems larger than few hundred atoms. In addition, the method does not include anharmonic effects and is only valid at low temperatures.

In this paper we report on calculations of free energy and vibrational entropy differences between ordered and compositional disordered $\mathrm{Ni}_{3} \mathrm{Al}$ in the temperature-range 200-1200 $\mathrm{K}$. The method employed in these calculations is based on the adiabatic switching method. ${ }^{12,13}$ The reversible path we have utilized directly connects the disordered system with the ordered one by switching both the interactions and the composition of the sites in the disordered system to those found in the ordered state. Finite temperature and anharmonic effects are included automatically in the moleculardynamics (MD) simulations. As we will show, anharmonic effects contribute significantly to the value of the vibrational entropy difference at high temperatures. Volume effects can be studied by carrying out simulations at constant volume and temperature (NVT) as well as at constant pressure and temperature (NPT). In addition, the vibrational spectra of individual atomic species in the alloy can be extracted from the simulations in a straightforward way. This information is extremely useful and very hard to obtain experimentally. For instance, in inelastic neutron scattering, the intensity of the Al spectra is suppressed considerably due to the smaller cross section of $\mathrm{Al}$ relative to the $\mathrm{Ni}$ atoms.

In addition, we present an alternative way of calculating the phonon DOS of the compositionally disordered system via the Fourier transform of the velocity-velocity autocorrelation function. Using this technique we calculated the phonon DOS of the disordered phase using systems with up to 11000 atoms in the computational cell. The disordered DOS is closer in shape to the DOS of the ordered $L 1_{2}$ phase than a fcc monoatomic DOS as expected from mean-field and virtual-crystal methods.

The atomic interactions are described by embedded-atommethod (EAM) potentials. ${ }^{14-16}$ EAM is a semiempirical formalism which has been used with great success in describing mechanical properties of metals and alloys. ${ }^{17}$ The potentials utilized in this work describe well the elastic properties and phonon dispersions of ordered $\mathrm{Ni}_{3} \mathrm{Al}$.

\section{METHODOLOGY}

\section{A. Potentials}

We have employed semiempirical embedded-atommethod (EAM) potentials ${ }^{14}$ to describe the atomic interactions in the Ni-Al system. Several potentials for the Ni-Al system have been developed. ${ }^{15,16,18-20}$ Here we have used a set of potentials developed for the full Ni-Al-H system. ${ }^{16}$ The potential has been applied successfully to the study of hydrogen interactions with $\mathrm{Ni}_{3} \mathrm{Al}$ grain boundaries and with $\mathrm{Ni} / \mathrm{Ni}_{3} \mathrm{Al}$ interfaces. ${ }^{16}$ The potential energy of the system is given by the expression

$$
E=\sum_{i=1}^{N}\left\{F_{t_{i}}\left(\rho_{t_{i}}\right)+\sum_{j \neq i} \phi_{t_{i}, t_{j}}\right\}
$$

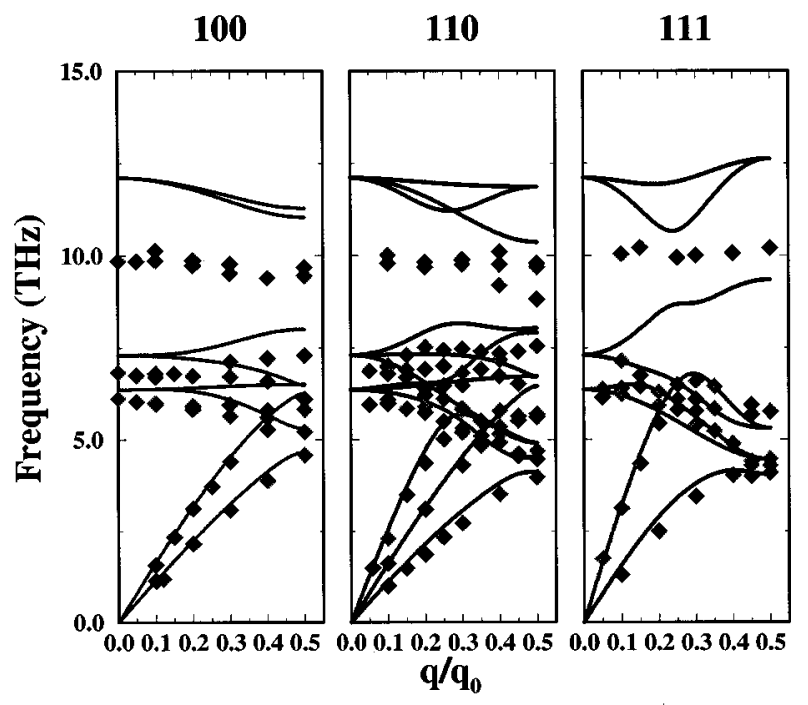

FIG. 1. Phonons dispersions of the $L 1_{2}$ ordered phase of $\mathrm{Ni}_{3} \mathrm{Al}$. Wave vector $q$ is given in units of $q_{0}=(2 \pi / a)$, where $a=$ lattice constant. Experimental points taken from the work of Stassis et al. (Ref. 21).

where $t_{i}$ is the atom type and the electron density at site $i$ is given by

$$
\rho_{t_{i}}=\sum_{j} f_{t_{j}}\left(r_{i j}\right)
$$

$f_{t_{j}}\left(r_{i j}\right)$ is the contribution to the electron density of atom $i$ due to atom $\mathrm{j}$ of type $t_{j}$.

The functional forms of these functions have been published elsewhere ${ }^{16}$ and will not be given here. The functions successfully describe the mechanical properties of $\mathrm{Ni}_{3} \mathrm{Al}$ and NiAl. Figure 1 shows the phonon dispersions of $L 1_{2} \mathrm{Ni}_{3} \mathrm{Al}$. The highest optical mode is about $25 \%$ higher than the experimental values, but overall the agreement is quite good considering that the bulk phonon dispersions were not used in the fit. Table I gives a comparison between experimental and calculated values of various mechanical quantities.

\section{B. Simulations and free-energy calculations}

The systems used in the calculations comprised up to 55000 atoms, although most of the calculations reported here were done with samples of 1372 and 10976 atoms. The compositionally disordered configuration was created by randomly interchanging $\mathrm{Al}$ and $\mathrm{Ni}$ types in the ordered $L 1_{2}$ structure, this amounts to randomly occupying the sites of a fcc crystal with the stoichiometry of $\mathrm{Ni}_{3} \mathrm{Al}$. The disordered phase thus created corresponds to the infinite temperature limit with no short-range order. No long-range compositional fluctuations are introduced in this randomization, but we believe this introduces very small errors in the free-energy difference between the ordered and disordered phases. Both the ordered and disordered samples were equilibrated over 5-10 ps using molecular dynamics at constant pressure and temperature ${ }^{29-31}$ (NPT) in order to obtain the correct volume at the various sampled temperatures: $200-1200 \mathrm{~K}$. No diffusion of atoms between lattice sites was observed during the equilibration and switching runs at any of the sampled tem- 
TABLE I. Calculated structural and elastic properties compared with experimental or first-principles values (in parentheses). The elastic constants for $\mathrm{Ni}_{3} \mathrm{Al}$ and all properties for $\mathrm{NiAl}$ are predicted while the remaining entries are fitted to experiment. Entries with asterisk are reproduced exactly by construction.

\begin{tabular}{lccccc}
\hline \hline & $a_{0}(\AA)$ & $E_{s}(\mathrm{eV} /$ atom $)$ & $C_{11}(\mathrm{GPa})$ & $C_{12}(\mathrm{GPa})$ & $C_{44}(\mathrm{GPa})$ \\
\hline $\mathrm{Ni}$ & $(3.52)^{* \mathrm{a}}$ & $(4.45)^{* \mathrm{~b}}$ & $246.4(246.5)^{\mathrm{c}}$ & $147.3(147.3)^{\mathrm{c}}$ & $124.8(124.7)^{\mathrm{c}}$ \\
$\mathrm{Al}$ & $(4.05)^{* \mathrm{a}}$ & $(3.36)^{* \mathrm{~b}}$ & $113.9(114.0)^{\mathrm{c}}$ & $62.0(61.9)^{\mathrm{c}}$ & $31.5(31.6)^{\mathrm{c}}$ \\
$\mathrm{Ni}_{3} \mathrm{Al}$ & $3.568(3.56)^{\mathrm{d}}$ & $4.598(4.57)^{\mathrm{e}}$ & $216(230)^{\mathrm{f}}$ & $155(149)^{\mathrm{f}}$ & $124(132)^{\mathrm{f}}$ \\
$\mathrm{NiAl}$ & $2.863(2.88)^{\mathrm{d}}$ & $4.425(4.51)^{\mathrm{e}}$ & $230(205)^{\mathrm{g}}$ & $161(142)^{\mathrm{g}}$ & $155(122)^{\mathrm{g}}$ \\
\hline \hline
\end{tabular}

${ }^{\text {a }}$ Reference 22.

${ }^{\mathrm{b}}$ Reference 23 .

${ }^{\mathrm{c}}$ Reference 24 .

${ }^{\mathrm{d}}$ Reference 25 .

${ }^{\mathrm{e}}$ Reference 26.

${ }^{\mathrm{f}}$ Reference 27.

${ }^{\mathrm{g}}$ Reference 28 .

peratures and the state of configurational disorder was preserved (no structural disorder was observed).

There are several approaches to the calculation of free energies and entropies of solids. A common technique of calculating entropies is via the density of states (DOS) which provides a way of counting microscopic states of the system.

$$
S_{\mathrm{vib}}=k_{B} \sum_{i}^{3 N} \ln \left(\omega_{i}\right)
$$

In the present work, we have calculated the free-energy and vibrational entropy differences between the ordered and disordered states as functions of temperature using adiabatic switching in a molecular-dynamics (MD) framework. This method, unlike quasiharmonic approximation methods is not restricted to low temperatures and is thus applicable over a wider temperature range.

The adiabatic switching formalism is based on Kirkwood's idea of connecting through a reversible path, the system of interest with a model system whose free energy is known. ${ }^{12}$ An effective Hamiltonian is constructed from both systems such that

$$
H_{\text {eff }}(\lambda)=(1-\lambda) H_{0}+\lambda H_{1}=H_{0}+\lambda \Delta H,
$$

where $H_{0}$ is the Hamiltonian of the atomistic system of interest, $H_{1}$ is the Hamiltonian of the model or reference system and $0 \leqslant \lambda \leqslant 1$. The free-energy difference can then be written as the integral over the thermodynamic path connecting both systems:

$$
\Delta F=F_{1}-F_{0}=\int_{0}^{1} \frac{\partial H_{\mathrm{eff}}(\lambda)}{\partial \lambda} d \lambda=\int_{0}^{1}\langle\Delta H\rangle_{\lambda} d \lambda,
$$

provided the path from $\lambda=0$ to $\lambda=1$ is reversible. The drawback with this approach (also referred to as $\lambda$ dynamics) is that several simulations for different values of $\lambda$ are necessary in order to evaluate the integral. Watanabe and Reinhardt suggested the integral be carried out dynamically. ${ }^{13}$ This is accomplished by allowing $\lambda$ to be a function of time. The actual time dependence of the parameter $\lambda$ is not important provided the time dependence is sufficiently slow that quasiergodicity holds and time averages become equivalent to quasistatic phase (ensemble) averages. ${ }^{32}$ In our calculations, we allow $\lambda$ to vary continuously over a time interval $\tau$ :

$$
\lambda(t)=\frac{t}{\tau}, \quad 0 \leqslant t \leqslant \tau .
$$

The free-energy difference is then computed from the MD time average of $\Delta H$ over the time interval $\tau$ :

$$
\Delta F=F_{1}-F_{0}=\frac{1}{\tau} \int_{0}^{\tau} \Delta H d t=\left\langle H_{1}\right\rangle-\left\langle H_{0}\right\rangle,
$$

where \langle\rangle means MD time averaging.

In the calculation of free energies of solids, the reference state $H_{0}$ is usually taken to be an Einstein (harmonic) solid. In our calculations, we selected a path which directly connects the disordered phase to the ordered one by switching the atomic types, masses, and interactions at each site to those found in the ordered state. This is accomplished by taking advantage of the fact that the disordered state is compositional and not topological disordered. Thus one can switch the types at each site in the disordered configuration to the appropriate atomic type which is found at that site in the ordered configuration. If the atomic type at a given site in the disordered state is different from the atomic type found at that site in the ordered phase, then we adiabatically switch the interactions and mass of the atom at that site to the interactions and mass of the appropriate type found in the ordered state. A similar path has been employed in the calculations of free energies of antiphase boundary defects in $\mathrm{Ni}_{3} \mathrm{Al}$ (Ref. 33) in which it was termed adiabatic "alchemy." An advantage of using this path over switching to a harmonic solid is in a higher accuracy of the results for relatively shorter switching times as well as a reduction in the number of switching runs since we do not need to compute the free energy of the ordered and disordered phases separately.

The switching runs were carried out at constant temperature and volume (NVT) and at constant pressure and temperature (NPT). In the present work we set the external pressure to zero in the NPT runs. The NPT calculations give the Gibbs free-energy difference between the disordered and ordered phases. The NVT runs give the Helmholtz free-energy 
difference. We found that switching over times of 10 ps gave an accuracy of about $10^{-4} \mathrm{eV} / \mathrm{atom}$ in the free-energy difference between ordered and disordered phases $(\Delta F)$. Using this technique, the free-energy difference was calculated every $100 \mathrm{~K}$ between 200 and $1200 \mathrm{~K}$ with systems of 1372 and 11000 atoms and at $300 \mathrm{~K}$ with a 55000 -atom system.

The free-energy difference was also computed from the integration of the internal energy difference between the ordered and disordered phases employing the thermodynamic relation

$$
\frac{\Delta F(T)}{T}=\frac{\Delta F\left(T_{0}\right)}{T_{0}}-\int_{T_{0}}^{T} \frac{\Delta E}{T^{2}} d T,
$$

with values of $\Delta F\left(T_{0}\right)$ at $T_{0}$ obtained from switching runs at $200 \mathrm{~K}$. The internal energy as a function of temperature was obtained by applying a constant heating rate $(\eta)$ to the ordered and disordered samples. A constant heating rate is applied by modifying the velocity of the particles at each time step: $\mathbf{v}^{\prime}=(\eta / \xi) \mathbf{v}$, where

$$
\xi=\frac{1}{3 N} \sum \mathbf{v} \cdot \mathbf{F}, \quad \eta=\frac{d T}{d t}=\frac{\left(T-T_{0}\right)}{\tau} .
$$

In above equations, $\tau$ is the simulation time in which the sample goes from an initial temperature $T_{0}$ to a final temperature $T$. We employed simulation times of about $35 \mathrm{ps}$ to go from 200 to $1200 \mathrm{~K}$.

The switching results from the 11000 -atom simulations are shown in Fig. 2(a). The free-energy difference obtained from integrating the internal energy difference [Eq. (8)] is shown by a dashed line. The integration of the internal energy gives a higher value of the free-energy difference at high temperatures than the discrete switching runs, but overall there is agreement within $0.5 \mathrm{meV} /$ atom.

In order to calculate the vibrational entropy difference, we fitted the free-energy difference data to a nonlinear curve using a least-squares procedure. The resulting nonlinear curve is

$$
\begin{aligned}
\Delta F_{\text {fit }}(T)= & 0.089869+2.2735 \times 10^{-5} T-5.041 \\
& \times 10^{-6} T \ln (T)-3.05 \times 10^{-10} T^{2},
\end{aligned}
$$

in units of eV/atom and with a root-mean-square ( $\mathrm{rms}$ ) deviation from the data of $1 \times 10^{-7}$. The solid line in Fig. 2(a) corresponds to the fit to the discrete data. The vibrational entropy was then calculated by taking the derivative with respect to temperature: $\Delta S=-\left(\partial \Delta F_{\mathrm{fit}} / \partial T\right)$.

\section{RESULTS}

The free energy of the disordered phase is higher than the ordered phase at all sampled temperatures. From our calculations, the difference in the free energy (this difference only includes the vibrational part of the entropy), $\Delta F$ decreases with temperature from about $0.09 \mathrm{eV} /$ atom at $200 \mathrm{~K}$ to about $0.07 \mathrm{eV} /$ atom at $1200 \mathrm{~K}$. Extrapolation of the $\Delta F_{\text {fit }}$ curve from Eq. (9), corrected to include the configurational entropy difference $\left(\Delta S_{\text {conf }}=0.56 k_{B} /\right.$ atom $\left.^{2}\right)$ gives $\Delta F_{\text {fit }}=0$ at 1435 $\mathrm{K}$. Experimentally, $\mathrm{Ni}_{3} \mathrm{Al}$ is known to remain ordered up to temperatures very close to its melting point. ${ }^{34,35}$ The critical order-disorder temperature of $\mathrm{NiAl}-\mathrm{Fe}$ alloys have been mea-

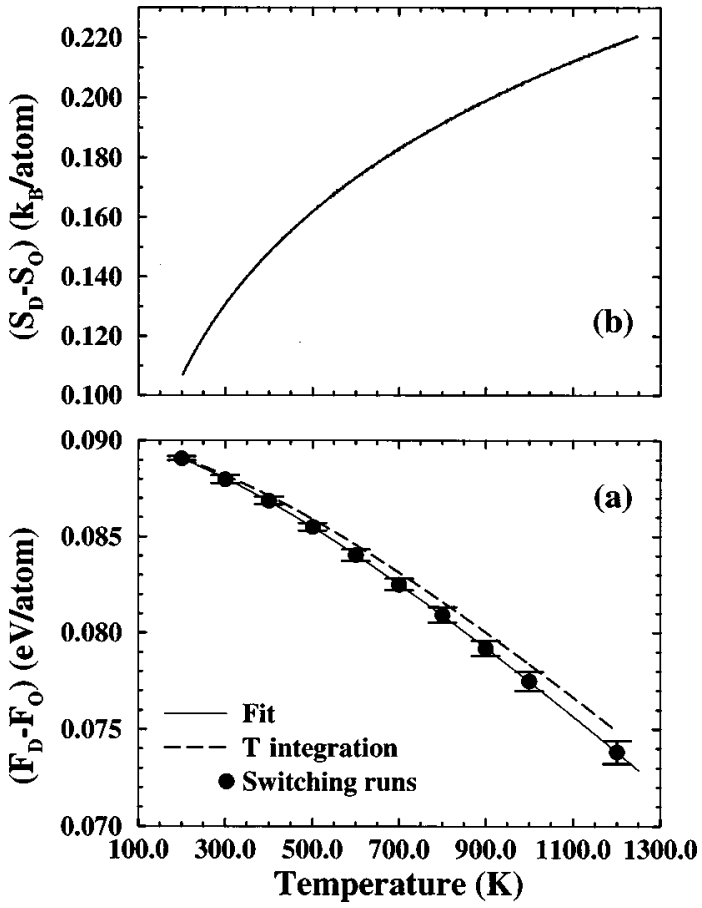

FIG. 2. (a) Free-energy difference between disordered and ordered $\mathrm{Ni}_{3} \mathrm{Al}$. Discrete points are from switching runs at constant temperature and pressure (NPT). The solid line is a nonlinear leastsquares fit with an $\mathrm{rm}$ of $1 \times 10^{-7}$. The dashed line represents the free-energy difference obtained from integrating the internal energy difference [Eq. (8)] with the value at $200 \mathrm{~K}$ obtained from NPT switching runs. (b) Vibrational entropy difference calculated from constant pressure simulations $(p=0)$.

sured and extrapolated to zero Fe concentration to give an order-disorder temperature of the $L 1_{2}$ and fcc phase of 1723 $\mathrm{K} .{ }^{36}$ We find that there exists a narrow temperature region within which $\mathrm{Ni}_{3} \mathrm{Al}$ disorders prior to melting.

Figure 2(b) shows the vibrational entropy difference as a function of temperature, obtained from the derivative to the fit to the free energy difference $\left[\Delta S=-\left(\partial \Delta F_{\text {fit }} / \partial T\right)\right]$. The vibrational entropy difference increases with temperature from about $0.12 k_{B}$ /atom at $200 \mathrm{~K}$ to $0.22 k_{B} /$ atom at 1200 $\mathrm{K}$. Our estimation of the error over this temperature interval is of about $\pm 0.005 k_{B}$ /atom.

In order to study size effects, we performed simulations with 1372, 11000 , and 55000 , atoms. The 55000 atoms simulations were limited to a temperature of $300 \mathrm{~K}$. Volume relaxation plays the most important role in the difference in entropy values. Our results as functions of system size are summarized in Table II. Our values at $300 \mathrm{~K}$ are in reasonable agreement with the recent experimental work of Fultz

TABLE II. Free energy, vibrational entropy, and volume differences between disordered and ordered $\mathrm{Ni}_{3} \mathrm{Al}$ as a function of system size $(N)$ (all data are at a temperature of $300 \mathrm{~K}$ ).

\begin{tabular}{lccc}
\hline \hline$N$ & $\Delta V(\%)$ & $\Delta F(\mathrm{eV} /$ atom $)$ & $\Delta S\left(k_{B} /\right.$ atom $)$ \\
\hline 1372 & 0 & 0.0846 & 0.06 \\
1372 & +0.9 & 0.0842 & 0.137 \\
10976 & +0.96 & 0.0879 & 0.139 \\
55296 & +1.1 & 0.0833 & 0.141 \\
\hline \hline
\end{tabular}




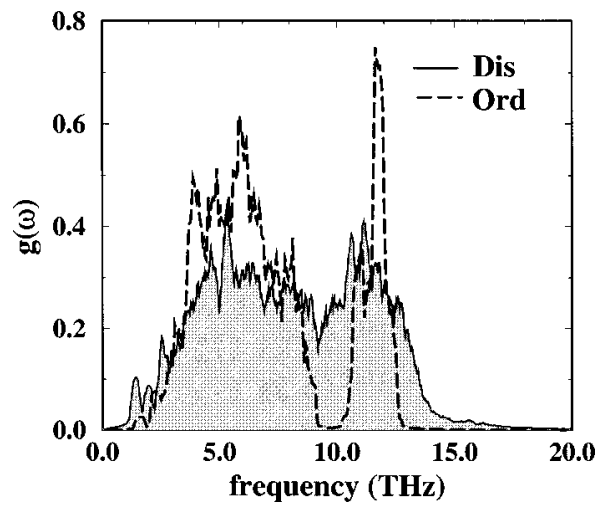

FIG. 3. Phonon DOS of $\mathrm{L}_{2}$ ordered (dashed curve) and disordered (shaded curve) $\mathrm{Ni}_{3} \mathrm{Al}$.

et al., which estimate $S_{\mathrm{vib}}^{\mathrm{dis}}-S_{\mathrm{vib}}^{\mathrm{ord}}=(+0.2 \pm 0.1) k_{B} /$ atom, ${ }^{5}$ but are smaller than the theoretical lattice-dynamics studies of Ackland which give $S_{\mathrm{vib}}^{\mathrm{dis}}-S_{\mathrm{vib}}^{\text {ord }}=0.29 k_{B} /$ atom. ${ }^{4}$ The 11000 and 1372 atom simulations results (free energy and vibrational entropy difference as a function of temperature) agree well with each other and we do not see a systematic difference (higher or lower) between them.

\section{A. Vibrational differences}

It has been proposed that this vibrational entropy difference could be due to a higher density of high-frequency modes (optical modes frequencies) in the vibrations of the $\mathrm{Al}$ atoms in the ordered phase relative to the disordered phase and the existence in the latter phase of a higher density of states at low frequencies. The difference in high-frequency modes will contribute more at higher temperatures. However, recent neutron-scattering experiments suggest that the vibrations in the disordered phase exhibit some intensity in the optical modes energies. ${ }^{5}$ We have investigated the difference in the vibrational spectra of disordered and ordered phases by computing the phonon density of states (DOS) of both ordered and disordered phases as well as the partial spectral intensity along the $\langle 100\rangle$ direction. While the phonon DOS of the ordered phase can be calculated via lattice dynamics, ${ }^{9}$ the disordered phase presents a problem for lattice-dynamics approaches due to the lack of periodicity. The approximate DOS of the disordered phase can be computed from the Fourier transform of the velocity-velocity autocorrelation function ${ }^{37}$

$$
g(\omega)=\int d t e^{i \omega t} \frac{\langle v(t) v(0)\rangle}{\left\langle v(0)^{2}\right\rangle} .
$$

Employing this technique we calculated the DOS of disordered $\mathrm{Ni}_{3} \mathrm{Al}$ employing 1372 and 11000 atom systems. The simulations were carried out at temperatures in the 10$150 \mathrm{~K}$ range and for time scales long enough to get a frequency resolution of $0.01 \mathrm{THz}$. The ordered (dashed curve) and disordered (shaded curve) $\mathrm{Ni}_{3} \mathrm{Al}$ DOS are shown in Fig. 3. The disordered DOS looks like a broadened version of the ordered DOS but more importantly, it looks very different from the DOS of a fcc monoatomic crystal casting doubts in the application of the virtual-crystal approximation to the analysis of the phonon DOS of this system.

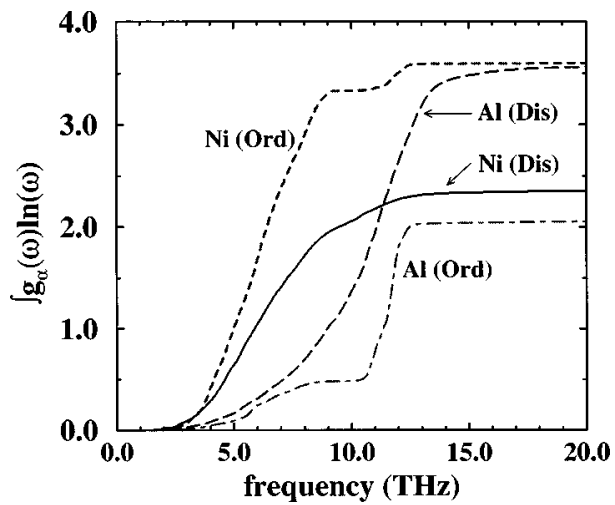

FIG. 4. Integrated partial DOS of $\mathrm{L}_{2}$ ordered and disordered $\mathrm{Ni}_{3} \mathrm{Al}$ weighted by $\ln (\omega)$. This quantity is related to the vibrational entropy contribution at high temperatures (in units of $k_{B} /$ atom).

Comparison of the partial DOS of the ordered and disordered phases shows the Al spectrum is more affected (broadens more) by compositional disorder than the Ni spectrum. This is certainly due to a larger change in the environment of the $\mathrm{Al}$ atoms than in the $\mathrm{Ni}$ atoms caused by compositional disorder. In Fig. 4 we have attempted to quantify the changes in the spectra of the $\mathrm{Al}$ and $\mathrm{Ni}$ due to compositional disorder by plotting the integrated partial DOS of the species weighted by $\ln (\omega)$. Above $7 \mathrm{THz}$, the partial DOS of $\mathrm{Al}$ in the disordered matrix is much larger than the one in the ordered matrix. At the same time, the cutoff frequency in the partial DOS of the disordered $\mathrm{Al}$ is just slightly higher (about $1 \mathrm{THz}$ ) than the ordered phase value.

We investigated further the vibrational spectra differences by computing the partial phonon spectral intensity along the $\langle 100\rangle$ direction. This is done by calculating the Fourier transform of the $q$-dependent velocity-velocity autocorrelation function:

$$
g_{\alpha \alpha}(\omega, \mathbf{q})=\int d t e^{i \omega t} \frac{\left\langle v_{\alpha}(\mathbf{q}, t) v_{\alpha}(-\mathbf{q}, t)\right\rangle}{\left\langle v_{\alpha}(\mathbf{q}, 0) v_{\alpha}(-\mathbf{q}, 0)\right\rangle},
$$

where

$$
v_{\alpha}(\mathbf{q}, t)=\frac{1}{N} \sum_{i=1}^{N} e^{i \mathbf{q} \cdot \mathbf{R}_{i}(t)} v_{i_{\alpha}}(t) .
$$

Figure 5 shows the partial spectral intensity along the $\langle 100\rangle$ direction, evaluated at two different $\mathbf{q}$ points: $|\mathbf{q}|$ $=0.125 q_{\max }\left[\right.$ Fig. 5(a)] and at $|\mathbf{q}|=\mathbf{q}_{\max }$ [Fig. 5(b)], $\mathbf{q}_{\max }$ $=(\pi / a)$. The plotted intensity represents the algebraic sum of the three polarizations: $g_{x x}+g_{y y}+g_{z z}$. We observe appreciable intensities in the disordered phase at optical modes frequencies of the ordered phase, especially at the end of the Brillioun zone $(\langle 100\rangle$ direction). Some intensity is also found at these high frequencies for the disordered phase at low values of $q$ [Fig. 5(a)]. We conclude that the vibrational spectrum of the disordered alloy is considerably more complex than can be predicted with a fcc unit cell.

The vibrational spectra of each type ( $\mathrm{Al}$ and $\mathrm{Ni}$ atoms) can also be calculated with this technique. Figure 6 shows the spectral intensity of the $\mathrm{Al}$ atoms in both the ordered (dashed) and the disordered (shaded) phases. In spite of the differences between the ordered and disordered alloys in lat- 


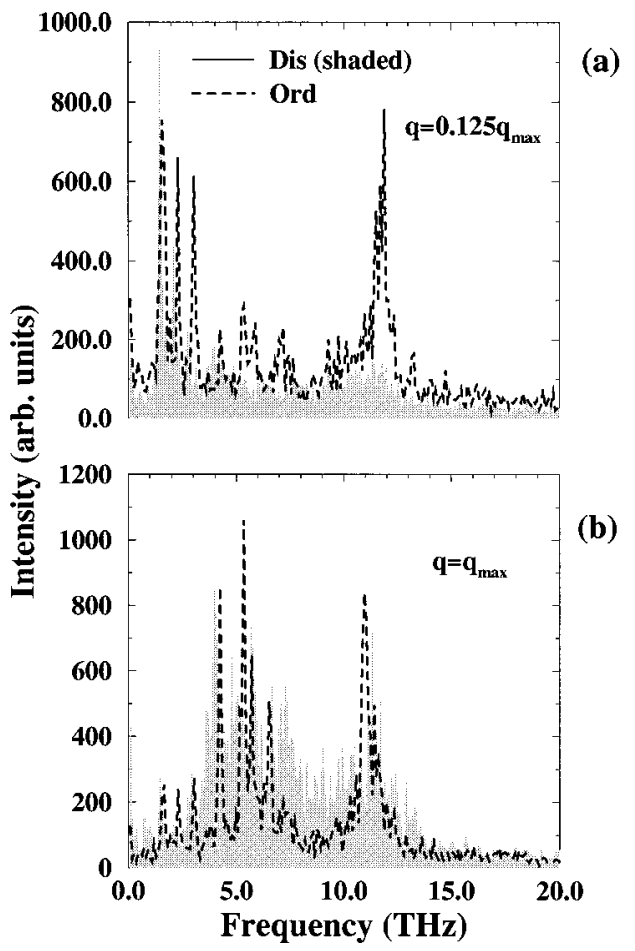

FIG. 5. Phonon spectral intensity of ordered (dashed) and disordered (shaded) $\mathrm{Ni}_{3} \mathrm{Al}$ at $2 q$ points: (a) $q=\left(0.125 q_{\max }\right)$ and (b) $q=q_{\max } \cdot q_{\max }=(\pi / a)$.

tice parameter and local atomic configurations, the cutoff frequencies for the $\mathrm{Al}$ atom vibrations are nearly the same in the two alloys. This can be seen more clearly in Fig. 4 which shows the integrated partial DOS weighted by the $\ln (\omega)$.

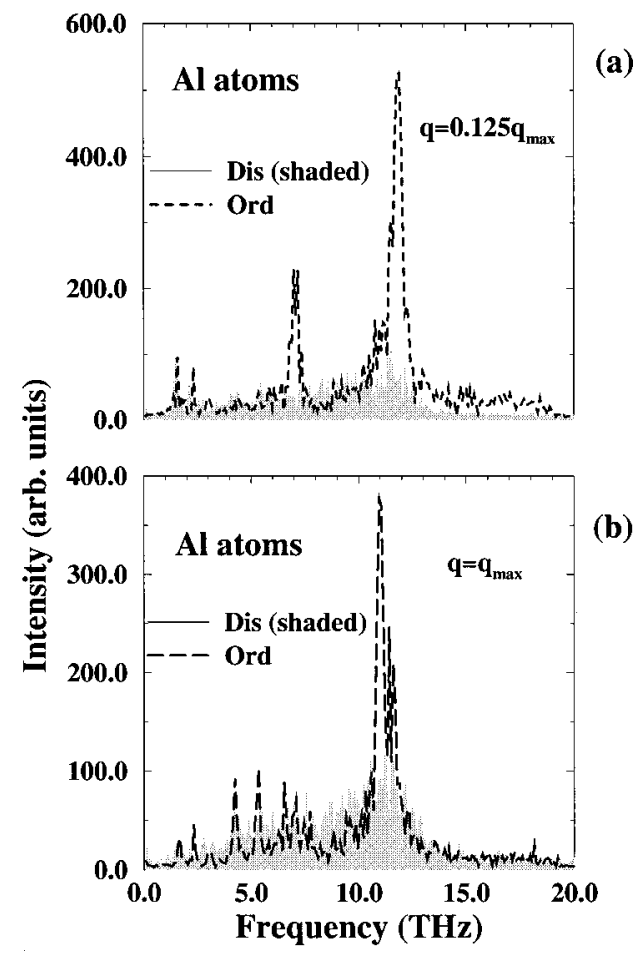

FIG. 6. Phonon spectral intensity of $\mathrm{Al}$ atoms in ordered (dashed) and disordered (shaded) $\mathrm{Ni}_{3} \mathrm{Al}$ evaluated at two different $q$ points: (a) $q=\left(0.125 q_{\max }\right)$ and (b) $q=q_{\max } \cdot q_{\max }=(\pi / a)$.

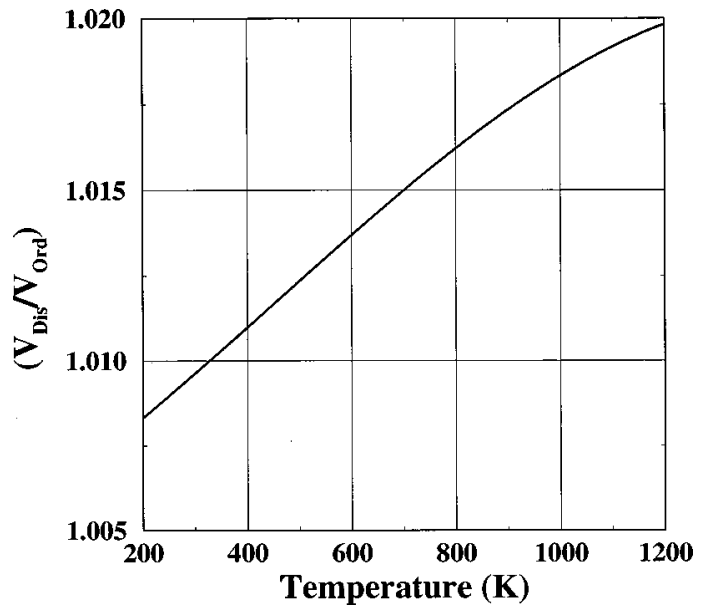

FIG. 7. Ratio of volume of disordered phase to the volume of ordered phase as a function of temperature.

Thus, we might conclude that there is no significant stiffening of the Ni-Al bonds in the ordered alloy.

\section{B. Volume effects}

We find that the vibrational entropy difference is very sensitive to volume changes. If the volume is not allowed to change, i.e., the switching is done at constant volume, then the vibrational entropy difference turns out to be much smaller $\left(0.06 k_{B}\right.$ /atom at $\left.300 \mathrm{~K}\right)$ with the disordered phase still having the higher entropy. (see Table II). This effect has also been reported by Ackland. ${ }^{4}$ The explanation given in the case of $\mathrm{Cu}_{3} \mathrm{Au}$, is that in the unrelaxed volume case, the $\mathrm{Au}-\mathrm{Au}$ bonds have shorter bonds which result in higher vibrational frequencies than in the relaxed case. $\mathrm{In} \mathrm{Ni}_{3} \mathrm{Al}$, we found that the disordered phase has a larger volume than the ordered phase. Figure 7 shows the ratio of the volumes in the two phases as a function of temperature. The difference is about $1-2 \%$ in the range $300-1200 \mathrm{~K}$, respectively. It is worth noting however, that preliminary experimental evidence shows that the lattice parameter of the disordered phase at room temperature is actually smaller (3.5632 $\AA$ ) than that of the ordered phase $(3.5792 \AA) .{ }^{38}$ Although comparable lattice parameters were obtained from disordered samples prepared by evaporation and by high-energy ball milling, the disordered samples may suffer uncertainties from the presence of defects such as vacancies. While the experimental data on heat capacity ${ }^{2}$ and phonon DOS (Ref. 5) were obtained from these same materials of $\mathrm{Ni}_{3} \mathrm{Al}$, there are other results in the literature for the difference in lattice parameter of the disordered and ordered $\mathrm{Ni}_{3} \mathrm{Al}$. These differences are not consistent with each other however, with the difference being reported as $0,{ }^{39}-0.2 \%,{ }^{40}+0.4 \%,{ }^{41}$ $+0.4 \%{ }^{42} \mathrm{We}$ also note that fcc disordered $\mathrm{Ni}_{3} \mathrm{Al}$ is ferromagnetic, whereas $L 1_{2}$ ordered $\mathrm{Ni}_{3} \mathrm{Al}$ is not. ${ }^{40,43}$

In order to gain more information on the nature of the vibrational difference, we also evaluated the root-meansquared (rms) displacement of the $\mathrm{Ni}$ and $\mathrm{Al}$ atoms in both ordered and disordered samples. We find that the largest difference comes from the $\mathrm{Al}$ atoms, which exhibit a larger amplitude in the disordered phase than in the ordered phase at all temperatures, but especially at low temperatures, where the $\mathrm{Al}$ atoms vibrate with an amplitude $20-10 \%$ higher than 


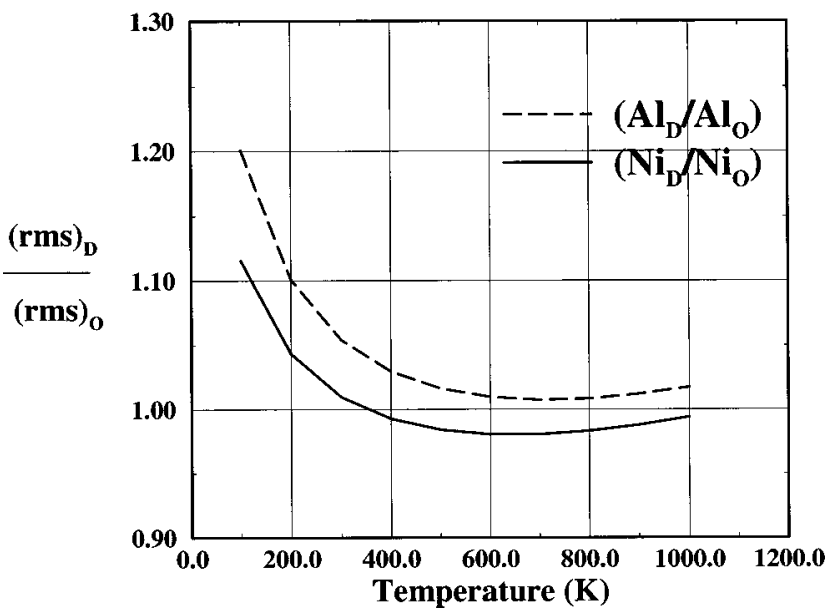

FIG. 8. Root-mean-squared (rms) displacement of $\mathrm{Al}$ and $\mathrm{Ni}$ atoms in both ordered and disordered phases as a function of temperature. The rms motion of the $\mathrm{Ni}$ atoms is larger in the ordered phase at temperatures above $350 \mathrm{~K}$.

in the ordered phase between 100 and $200 \mathrm{~K}$, respectively. This is shown in Fig. 8, where we have plotted the ratio of the rms displacement of the $\mathrm{Al}$ and $\mathrm{Ni}$ atoms in the disordered phase to that of the ordered phase as a function of temperature. This ratio decreases with temperature for both $\mathrm{Ni}$ and $\mathrm{Al}$ types. However, while the $\mathrm{Al}$ atoms in the disordered phase exhibit a larger rms displacement at all sampled temperatures, the $\mathrm{Ni}$ atoms have a larger amplitude of vibration above $400 \mathrm{~K}$ in the ordered phase. This amplitude difference is small, about $2 \%$ at temperatures above $400 \mathrm{~K}$.

\section{CONCLUSIONS}

We have studied in detail the vibrational and thermodynamic differences in $\mathrm{Ni}_{3} \mathrm{Al}$ in two different states of chemical order: in the ordered $L 1_{2}$ structure and in a fcc solid solution (compositional disordered phase). We have calculated the free energy and vibrational entropy difference employing the method of adiabatic switching in a moleculardynamics framework with a thermodynamical path which directly connects the disordered state with the ordered one.
The free-energy difference indicates that $\mathrm{Ni}_{3} \mathrm{Al}$ remains ordered up to temperatures close to its melting temperature. Our calculations predict the existence of a narrow temperature region $(1435-1640 \mathrm{~K})$ within which $\mathrm{Ni}_{3} \mathrm{Al}$ disorders prior to melting. This result is within $12 \%$ of experimental predictions from NiAl-Fe alloys. ${ }^{36}$ The vibrational entropy of the disordered state is larger than of the ordered state at all sampled temperatures with the difference increasing with temperature: $S_{\text {vib }}^{\text {dis }}-S_{\text {vib }}^{\text {ord }}=0.12-0.22 k_{B} /$ atom in the range 200-1200 K, respectively. This strong temperature dependence of $S_{\text {vib }}^{\text {dis }}-S_{\text {vib }}^{\text {ord }}$ suggests that differences in vibrational entropies of alloy phases obtained at low temperatures may be seriously in error when used for calculating phase boundaries at elevated temperatures.

The phonon density of states of the disordered phase was computed from the Fourier transform of the velocity-velocity correlation function. This method allows the use of a larger number of atoms in the computational cell than those employed in lattice-dynamics methods. The disordered DOS appear as a broadened version of the ordered DOS and are different in shape from a monoatomic fcc DOS as predicted by mean-field methods like the virtual-crystal approximation. Comparison of partial spectral intensities near the $\Gamma$ point and at end of the Brillioun zone along the $\langle 100\rangle$ direction show considerable intensities at optical mode frequencies of the ordered phase.

Finally, we find that volume effects play the most crucial role in the magnitude of the vibrational entropy difference. If the volume is not allowed to change, i.e., the volume difference between ordered and disordered phases is the same, the difference in vibrational entropy decreases considerably: $S_{\mathrm{vib}}^{\text {dis }}-S_{\mathrm{vib}}^{\text {ord }}=0.06 k_{B} /$ atom at $300 \mathrm{~K}$, compared with a value of $0.14 k_{B} /$ atom obtained from constant-pressure simulations at the same temperature.

\section{ACKNOWLEDGMENTS}

This work was supported by the U.S. Department of Energy under Contract No. DE-AC04-94AL85000. R.R. would like to thank Sandia National Laboratories, Livermore for their financial support during various stages of this work.
*Author to whom correspondence should be addressed.

${ }^{\dagger}$ Present address: Seagate Technology, Bloomington, MN 55420.

${ }^{1}$ S. J. Clark and G. J. Ackland, Phys. Rev. B 48, 10899 (1993).

${ }^{2}$ L. Anthony, J. K. Okamoto, and B. Fultz, Phys. Rev. Lett. 70, 1128 (1993).

${ }^{3}$ L. Anthony, L. J. Nagel, J. K. Okamoto, and B. Fultz, Phys. Rev. Lett. 73, 3034 (1994).

${ }^{4}$ G. J. Ackland, in Alloy Modeling and Design, edited by G. M. Stocks and F. E. A. Turchi (The Minerals, Metals, and Materials Society, Warrendale, PA, 1994), p. 149.

${ }^{5}$ B. Fultz, L. Anthony, L. J. Nagel, R. M. Nicklow, and S. Spooner, Phys. Rev. B 52, 3315 (1995).

${ }^{6}$ R. A. Oriani, Acta Metall. 4, 15 (1956).

${ }^{7}$ S. V. Radcliffe, B. L. Averbach, and M. Cohen, Acta Metall. 9, 169 (1961).

${ }^{8}$ H. J. Leamy, Acta Metall. 15, 1839 (1976), and references therein.
${ }^{9}$ A. A. Maradudin, E. W. Montroll, and G. H. Weiss, Theory of Lattice Dynamics in the Harmonic Approximation (Academic, New York, 1971).

${ }^{10}$ K. F. Stripp and J. G. Kirkwood, J. Chem. Phys. 22, 1579 (1954).

${ }^{11}$ P. J. Wojtowicz and J. G. Kirkwood, J. Chem. Phys. 33, 1299 (1960).

${ }^{12}$ J. G. Kirkwood, J. Chem. Phys. 3, 300 (1935).

${ }^{13}$ M. Watanabe and W. P. Reinhardt, Phys. Rev. Lett. 65, 3301 (1990).

${ }^{14}$ M. S. Daw and M. I. Baskes, Phys. Rev. B 29, 6443 (1984).

${ }^{15}$ M. I. Baskes, J. E. Angelo, and N. R. Moody, in Hydrogen Effects in Materials, edited by A. W. Thompson and N. R. Moody (The Minerals, Metals, and Materials Society, Warrendale, PA, 1996), p. 77.

${ }^{16}$ J. E. Angelo, M. I. Baskes, and N. E. Moody, Model. Simul. Mater. Sci. Eng. 3, 289 (1995).

${ }^{17}$ M. S. Daw, S. M. Foiles, and M. I. Baskes, Mater. Sci. Rep. 9, 251 (1993). 
${ }^{18}$ S. P. Chen, D. J. Srolovitz, and A. F. Voter, J. Mater. Res. 4, 62 (1989).

${ }^{19}$ V. Vitek, G. J. Ackland, and J. Cserti, in Alloy Stability and Design, edited by G. M. Stocks et al., MRS Symposia Proceedings No. 186 (Materials Research Society, Pittsburgh, 1991), p. 237.

${ }^{20}$ For a review or Ni/Al potentials see M. I. Baskes, Acta Metall. Sin. 8, 287 (1993).

${ }^{21}$ C. Stassis, F. X. Kayser, C. K. Loong, and D. Arch, Phys. Rev. B 24, 3048 (1981).

${ }^{22}$ C. S. Barret and T. B. Massalski, Structure of Metals (McGrawHill, New York, 1966).

${ }^{23}$ Metal Reference Book, edited by C. J. Smith, 5th ed. (Butterworths, London, 1976).

${ }^{24}$ G. Simmons and H. Wang, Single Crystals Elastic Constants and Calculated Aggregate Properties: A Handbook (MIT Press, Cambridge, MA, 1971).

${ }^{25}$ Smithells Metals Reference Book, edited by E. A. Brandes, 6th ed. (Butterworths, London, 1983).

${ }^{26}$ W. W. Wood, J. Chem. Phys. 52, 729 (1970).

${ }^{27}$ S. M. Foiles, M. I. Baskes, and M. S. Daw, Mater. Sci. Forum 37, 223 (1989).

${ }^{28}$ N. Rusovic and H. Warlimont, Phys. Status Solidi A 44, 609 (1977).

${ }^{29}$ S. Nosé, J. Chem. Phys. 81, 511 (1984).
${ }^{30}$ W. G. Hoover, Phys. Rev. A 31, 1695 (1985).

${ }^{31}$ W. G. Hoover, Phys. Rev. A 34, 2499 (1986).

${ }^{32}$ B. L. Holian, H. A. Posch, and W. G. Hoover, Phys. Rev. E 47, 3852 (1993).

${ }^{33}$ A. J. Skinner, J. V. Lill, and J. Q. Broughton, Model. Simul. Mater. Sci. Eng. 3, 359 (1995).

${ }^{34}$ C. L. Corey and B. Lisowsky, TMS-AIME 239, 239 (1967).

${ }^{35}$ F. J. Bremer, M. Beyss, and H. Wenzl, Phys. Status Solidi A 110, 77 (1988).

${ }^{36}$ R. W. Cahn, P. A. Siemers, J. E. Geiger, and P. Bardhan, Acta Metall. 35, 2737 (1987).

${ }^{37}$ J. M. Dickey and A. Paskin, Phys. Rev. 188, 1407 (1969).

${ }^{38}$ B. Fultz (unpublished).

${ }^{39}$ A. R. Yavari and B. Bochu, Philos. Mag. A 59, 697 (1989).

${ }^{40}$ K. Sumiyama, Y. Hirose, and Y. Nakamura, Phys. Rev. A 114, 693 (1989).

${ }^{41}$ G. F. Zhou, M. J. Zwnenburg, and H. Bakker, J. Appl. Phys. 78, 3438 (1995).

${ }^{42}$ S. Gialanella, S. B. Newcomb, and R. W. Cahn, in Ordering and Disordering in Alloys, edited by A. R. Yavari (Elsevier, London, 1992), p. 67.

${ }^{43}$ A. R. Yavari, P. Crespo, E. Pulido, A. Hernando, G. Fillion, P. Lethuillier, M. D. Baro, and S. Surinach, in Ordering and Disordering in Alloys, edited by A. R. Yavari (Elsevier, London, 1992), p. 12. 